\title{
CORRECTION NOTE TO PREDICTION OF OUTSTANDING LIABILITIES IN NON-LIFE INSURANCE, $A B$ 23, 95-115
}

\section{RAGNAR NORBERG}

1. In (4.17) a factor $W$ is missing in the numerator of the expression in the middle.

2. The predictor of $X^{\text {orns }}$ proposed in Paragraph 5B is not unbiased in general as a simple counterexample will show. The proposed reserve on an individual orns claim with past history $T, U, V>v^{\prime}=\tau-T-U,\left\{Y^{\prime}\left(v^{\prime \prime}\right)\right.$; $\left.0 \leq v^{\prime \prime} \leq v^{\prime}\right\}$, is

$$
R=E\left[Y \mid T, U, Y>Y^{\prime}\left(v^{\prime}\right)\right]-Y^{\prime}\left(v^{\prime}\right) .
$$

Suppose that $Y=V$ and $Y^{\prime}\left(v^{\prime \prime}\right)=Q\left(v^{\prime \prime} / V\right) V, 0 \leq v^{\prime \prime} \leq V$, where $Q$ is a non-decreasing, deterministic function on $[0,1]$ such that $Q(0)=0$ and $Q(1)=1$. In this case $R=E\left[V \mid T, U, V>Y^{\prime}\left(v^{\prime}\right)\right]-Y^{\prime}\left(v^{\prime}\right)$. If $Q(s)>s$ for all $s \in(0,1)$, then $Y^{\prime}\left(v^{\prime}\right)=Q\left(v^{\prime} / V\right) V>v^{\prime}$, hence

$$
R \geq E\left[V \mid T, Y, V>v^{\prime}\right]-Y^{\prime}\left(v^{\prime}\right) .
$$

Now, the expression on the right here is an unbiased predictor, confer relation (4.3) and the related text in the follow-up paper Norberg (1999). Thus the proposed reserve is systematically too high. Similarly, if $Q(s)<s$ for all $s \in(0,1)$, then the proposed reserve is systematically too low.

My thanks are due to Svend Haastrup, who pointed out this problem. For a discussion by him, see Haastrup (1997).

\section{ADDITIONAL REFERENCES}

HaAstrup, S. (1997). Some fully Bayesian micro models for claims reserving. Ph.D. thesis, Laboratory of Actuarial Mathematics, University of Copenhagen.

NORBERG, R. (1999). Prediction of outstanding claims II: Model variations and extensions. ASTIN Bull. 29, No. 1, 5-25. 